\section{Free recall of object categories as a function of number of presentations of varied or repeated specimens*}

\author{
WALTER F. DAVES and ROGER C. RINN \\ Georgia State University, Atlanta, Ga. 30303
}

Photographic slides of 18 categories of common objects were presented to 24 male and 24 female $\mathrm{Ss}$, who were divided into four groups receiving one, two, three, or four separate presentations of each category. For half of the categories ( $V$ categories) up to four different specimens represented the category; for the other half ( $R$ categories) the same specimen was repeated up to four times. Recall was tested both immediately and after a delay of approximately 2 weeks. For immediate recall the $\mathrm{V}$ categories were recalled best, and recall was a positive function of number of presentations. The $R$ categories were recalled less after two and three presentations than after one, but recall increased with the fourth presentation. Similar findings were obtained in 2 -week recall, although there was no overall effect of variety, and interpretation of the findings was complicated by significant two-way interactions between sex and variety and sex and repetitions, as well as a significant main effect of sex.

Several recent experiments have explored the role of stimulus variation in learning and recall. When the task is free recall of categories of stimuli, Ss have been found to show superior recall for those categories that are represented by several different specimens rather than the same specimen repeated an equivalent number of times. Such has been the case for pictures of common objects (Bevan, Dukes, \& Avant, 1966; Daves \& Adkins, 1969), as well as for nouns modified by several different adjectives or the same adjective repeated (Bevan et al, 1966; Bevan \& Dukes, 1967). Similar results were found for free recall of objects in 4and 5-year-old children (McCarson \& Daves, in press) and in college students, when the stimuli were nonsense forms and a recognition measure of memory was used (Avant \& Bevan, 1968). In the latter experiment it was pointed out that too much variation might result in a decrement in performance.

One question arising out of the above findings is whether the effect is one of enhancement of performance due to variety or inhibition of performance due to repetition. While there is no need to document the fact that, in most learning situations, repetition enhances performance, there are specific instances in which repetition has been shown to interfere with recall. Jahnke (1969), for

* This research was partly supported by funds from the National Science Foundation. Grant No.GU 2751, to Georgia State University. Requests for reprints should be directed to Dr. Walter F. Daves, Department of Psychology, Georgia State University, 33 Gilmer Street. S.E.. Atlanta, Georgia 30303 . example, has dealt with the Ranschburg effect, in which a repeated digit in a memory-span task is often not recalled. A similar phenomenon is the "prefix effect" (e.g., Crowder \& Hoenig, 1969), in which a redundant digit, not part of the string of digits to be recalled, reduces the observed digit performance occurred under a variety of conditions, including when the S had to repeat the first digit of the string. Thus, there is some rationale for exploring the role of repetition in memory for pictorial material.

Specifically, the present study compares recall of categories of common objects when the categories were repeated one, two, three, or four times with either the same object or different objects representing the category

\section{STIMULUS MATERIAL}

Color slides of 18 categories of objects were prepared, with nine categories assigned to each of two groups: V(aried) and R(epeated). For the $V$ categories there was a slide for each of four different specimens of that category (e.g., for the category "watch" there were four different watches). The remaining $V$ categories were: typewriters, flowers, knives, candles, pillows, bottles, balls, and purses. For the $\mathrm{R}$ categories, four identical slides of each specimen were used. The $\mathrm{R}$ categories included rings, keys, pens, chairs, cereal boxes, ash trays, shoes, lamps, and books. These objects were assigned to the $\mathrm{V}$ or $\mathrm{R}$ categories on the basis of other work ${ }^{1}$ in our laboratory which had suggested that the two groups would produce approximately equivalent recall. All objects were photographed against a common off-white background. The span of the $S$. The reduction in slides were projected with a Kodak Carousel projector, which was modified so that exposure duration could be controlled by means of a solenoid latch attached to the internal shutter. Stimulus duration was $0.5 \mathrm{sec}$, and the interval between onsets of adjacent slides was $2.0 \mathrm{sec}$. These intervals were controlled by a Hunter Model 1514 timer and were checked periodically with a Tektronix No. 503 oscilloscope driven by a silicon photocell located at the center of the screen on which the slides were projected.

\section{SUBJECTS AND DESIGN}

The $\mathrm{Ss}$ were 48 students in introductory psychology classes at Georgia State University who volunteered for the experiment to meet a requirement for the course. They were tested in groups varying in size from one to six. Each group of Ss was assigned to one of four repetition groups (Groups 1, 2, 3, and 4), with Group 1 receiving one presentation of each of the categories to be recalled, Group 2 receiving two presentations, etc. For Groups 2, 3, and 4, half of the categories were represented by varied specimens, and half were represented by the same specimen repeated. Ultimately, six males and six females were assigned to each group. Thus, in the design, repetition and sex were between-S variables, and variety level was manipulated within Ss.

\section{PROCEDURE}

The Ss, upon entering the laboratory, were seated in desk chairs and given writing pads and pencils. Instructions were read aloud as follows: "This is a study of visual perception. I'm going to show you some pictures of objects. Pay close attention to each individual object. After you have seen them all, I'll ask you to recall the objects. Please don't talk or write during the session."

The lights were turned off and, after being shown the slides, Ss were given 2 min to write down as many of the objects as they could remember. They were then given a sheet of paper with addition problems and asked to begin adding up the columns of figures. After $1 \mathrm{~min}$, a second recall trial was run for $2 \mathrm{~min}$. Following another $1 \mathrm{~min}$ of addition, a third recall trial was run.

The Ss in Repetition Group 1 viewed 18 slides, one from each of the 18 categories; Group 2 viewed 36 slides, two from each category, with the same specimen repeated for each of the $R$ categories and two different specimens for each of the $\mathrm{V}$ categories. Group 3 viewed 54 slides, with three identical Rs and three different $V_{s}$; Group 4 viewed 72 slides, with four identical Rs and four different Vs. In all cases the slides were haphazardly 


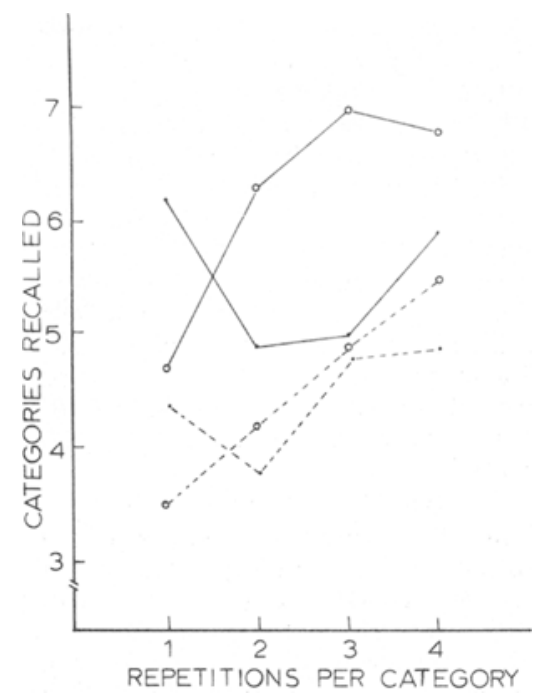

Fig. 1. The Variety by Repetitions interactions for immediate (solid lines) and two-week (dashed lines) recall. The open circles indicate recall of the $V$-categories, the dots recall of the $R$-categories.

arranged in blocks of 18 , so that each block contained one specimen from each category.

After a 2-week interval, the $\mathrm{E}$ went to the Ss' classrooms and asked them to recall as many of the objects as they could. Since all Ss were in two classes and the experiment required approximately 1 week to run, the actual retention interval varied from 2 to 3 weeks for individual Ss. RESULTS

The recall list of each $\mathbf{S}$ was scored for category recall. Thus, if he recalled at least one item from a given category, he was given credit for that category. Only data from immediate and 2-week recall are presented, since inspection revealed no essential differences in the results between the immediate and the two delayed recall conditions. These results are presented in Fig. 1 (solid lines). Analysis of variance of the immediate recall indicated a significant Variety by Repetitions interaction $(\mathrm{F}=\mathbf{1 0 . 1 1}$, $\mathrm{df}=3 / 40, \quad \mathrm{p}<.005)$. Orthogonal polynomials tests applied to the simple effects of repetition indicate that recall increased linearly as a function of repetitions for the $V$, but not the $R$, categories $(F=19.88)$. The quadratic component was, however, significant for both $V \quad(F=15.54)$ and $R$ $(F=9.80)$ categories. The inhibitory effect for the $R$ categories was overcome by the fourth repetition and, at the same time, there was a leveling off of the increment due to variety. There was, in addition, a significant main effect of variety level $(\mathrm{F}=6.45, \mathrm{df}=1 / 40, \mathrm{p}<.025)$. The main effect of repetitions was not significant $(F=1.39)$.

The 2 -week recall data are shown in Fig. 1 (dashed lines). Unfortunately, four of the Ss were not available for testing. Three of the Ss were females in Group 2, and one was a male in Group 4. Values for the missing cells were estimated by determining the overall mean decrement in recall between the immediate and the 2 -week tests, and applying this correction to the Ss' scores in immediate recall. One degree of freedom was then subtracted for each of the eight values (one $R$ and one $V$ score for each $\mathrm{S}$ ). The main effect of repetitions was significant $(F=18.29$, df $=3 / 36, p<.005$ ), as was the main effect of sex $(F=5.88, \quad d f=1 / 36$, $\mathrm{p}<.025$ ). Thus, overall recall increased with repeated presentations of the stimuli (Fig. 1), and recall for the females was higher than for the males (9.4 vs 8.7 categories recalled). As in immediate recall, the Variety by Repetitions interaction was significant $(F=5.24, \quad d f=3 / 36, \quad p<.005)$, and there was a significant linear trend for both $V(F=13.08)$ and $R(F=8.39)$ categories. The quadratic component was not significant for either set of categories, although the quadratic F for the $R$ categories was 4.08 , which is only .02 shy of the critical value for the .05 level and would have been significant had not the degrees of freedom been subtracted for the estimated data.

The above effects are qualified by significant interactions between sex and variety $(\mathrm{F}=13.72, \quad \mathrm{df}=1 / 36$, $\mathrm{p}<.005)$ and between sex and repetitions ( $\mathrm{F}=3.25, \quad \mathrm{df}=3 / 36$, $\mathrm{p}<.05)$. Tests of the simple effects of variety for males and females indicate that the males recalled 4.6 of the $R$ but only 4.1 of the $V$ categories $(\mathrm{F}=5.88, \mathrm{df}=1 / 36, \mathrm{p}<.025)$, while the females recalled 5.0 of the $\mathrm{V}$ and 4.4 of the $R$ categories ( $F=7.84$, $\mathrm{df}=1 / 36, p<.025)$. Simple effects of the Sex by Repetitions interaction indicate that both males and females increased recall as a function of repetitions $[F($ males $)=12.41$, $\mathrm{df}=3 / 36, \mathrm{p}<.005$; $F($ females $)=8.49, \quad d f=3 / 36$, $\mathrm{p}<.005]$. The interaction appears to be due primarily to a great improvement in recall by the males in Repetition Group 3.

\section{DISCUSSION}

The results clearly indicate that repetition of the same specimen decreased immediate recall of the $R$ categories, at least for repetition frequencies up to three. At the same time, recall of the $\mathrm{V}$ categories increased as a function of repetition. The curvilinear effect seen for the $R$ categories stigrests that at some point beyond four repetitions, the superiority of the $\mathrm{V}$ calegories would be reduced to zero or perhaps surpassed by the $\mathrm{R}$ categories. There is, of course, no direct test of this notion at the moment.

Although results for the 2 -week recall were complicated by missing data and apparent differences between males and females, the overall effect of variation and repetition appears similar to that found for immediate recall, in that two repetitions seemed to produce less recall of the $R$ categories than did one repetition. There was, however, no overall superiority of variety in the 2 -week recall. This reduction in the overall effect of variation in recall is in contrast to a report by Bevan et al (1966), who found an enhanced superiority of variety over the longer retention intervals (immediate, $2-4$ days, and 3 weeks), which was associated with increased forgetting of the repeated categories. Variety, however, was manipulated in their study as a between-Ss variable, and it is possible that this fact could in some way account for the difference. Perhaps there was associative facilitation between the varied and repeated categories such that the enhanced recall of the $\mathrm{V}$ material, over the longer interval, generalized to the other ( $R$ ) categories in the list. On the other hand, one cannot ignore the fact that 2-week recall was tested in the Ss' classrooms, whereas initial learning and recall were conducted in the laboratory in our study, and 8 of the 96 data points had to be estimated in the 2-weeks recall. Thus, any more definitive explanation of the differences must await further research.

Regardless of what happens after long retention intervals, the main phenomenon, superiority in recall for $V$ as opposed to $R$ categories, is yet to be explained. The inhibitory effect observed in the present experiment is perhaps due to the operation of a limited-capacity retrieval system which Tulving (1968) has proposed. If the $V$ categories are more available, and if, as Tulving suggests, "... retrieval of one unit makes access to another unit impossible even though the latter is available in storage [p. 27]," one might expect recall of the other categories to be inhibited. On the other hand, the increased accessibility of the $\mathrm{V}$ categories remains a problem. Perhaps the varied specimens provide more retrieval "tags," or perhaps the superordinate categorial process suggested by Bousfield \& Cohen (1955) is initiated by the $V$ but not by the $R$ categories, and this process is more available for recall. Further 
research shoud focus upon these possible explanations.

\section{REFERENCES}

AVANT, L L \& BEVAN, W. Recognition of a stimulus class member after training with varied numbers of cases per elass. Journal of General Psychology, 1968, 78 . 241-246.

BEVAN, W. \& DUKES, W. F. Stimulus-variation and recall: The role of belongingness. American Joumal of Psy chology, 1967, 80, 309-312.

BEVAN, W., DUKES, W. F., \& AVANT, L. $L$. The effect of variation in specific stimuli on memory for their superordinates. American Joumal of Superordinates. American Jo

BOUSFIELD, W. A., \& COHEN, B. H. The occurrence of clustering in the recall of randomly arranged words of different frequencies of usage. Journal of General Psychology, 1955, 52, 83-95.

CROWDER, R. G., \& HOENIG, Y.J. Intertrial competition and the prefix effect. Journal of Experimental Psychology, 1969, 79, 368-370.

DAVES, W. F. \& ADKINS, M. Stimulus variation and free recall: A confirmation American Journal of Psychology, 1969, 82, $122-124$
DAVES. H. F. \& RINN. R. C. The variety effect in free recall as a function of pre-recall activity. Psychonomic Science, $1971,22,226-227$

JAHNKE, J. C. The Ranschburg effect. Psychological Review, 1969, 76, 592-605. McCARSON, C., \& DAVES, IV. F. Free recall of object names in pre-school children as a function of intracategory children as a function of intracategory variation.

TULVING, E. Theoretical issues in free recall. In $T$. R. Dixon and D. L. Horton (Eds.), Verbal behavior and general behavior theory. Englewood Cliffs, N.J: Prentice-Hall, 1968. Pp. 2-36.

$$
\text { NOTE }
$$

1. Daves \& Rinn (1971); and Daves, IV. F. \& Rinn, R. C. The effect of stimulus duration and interstimulus interval on the superiority in recall of varied over repeated categories, in preparation. Both of the above studies show that the variety effect described in the present experiment occurs regardless of which particular categories of objects are repeated, as opposed to varied. Therefore, that control was not included in the present experiment, but rather an attempt was made to assign categories to the $R$ or the $V$ group so that overall recall would be approximately equal.

\section{Retrieval time in forward and backward recall*}

\section{TERRY R. ANDERS and TIMOTHY D. LILLYQUIST $†$}

Harvard Medical School, Boston, Mass. 02114

The present experiment was designed to compare the rates of forward and backward digit recall. The results show that recall was faster in forward order than in backward order. This finding is consistent with the hypothesis that information can be retrieved from short-term memory only in the same order in which it is stored.

Retrieval from short-term memory has been assumed by some to be unidirectional, i.e., information can be retrieved only in the same order in which it was stored (Broadbent, 1958; Conrad, 1965; Wickelgren, 1966; Yntema \& Trask, 1963). Evidence frequently cited in support of this contention is the superiority of the forward memory span over the backward span. It is maintained that the forward span is longer because information is retrieved from memory in the same order in which it is to be reported, while backward recall requires that the retrieved information be reordered before it can be reported. Conrad (1965) suggested that this transformation is accomplished by a succession of rapid to-and-fro scans. That is, each item reported in backward order is preceded by S's scanning forward through the list in memory to locate and retrieve it. This

*This research was supported by U.S. Public Health Service Grant No. MH-17110 and by Social Rehabilitation Service Grant No. RD-2685-M

+ Now at Northeastern University. Boston, Mass. 02115. additional operation requires time, over the course of which some forgetting would be expected to occur. As a result, the backward memory span is normally shorter than the forward span.

This account of the differences between the forward and backward memory spans predicts that, in addition to the forward span being longer, items recalled in forward order should also be reported at a faster rate. The forward memory span has been shown many times to be longer than the backward span. The rate at which the items are reported has not, however, previously been investigated. The present experiment was designed to compare the rates at which items are recalled under these two conditions and to further explore the underlying retrieval processes.

$$
\text { METHOD }
$$

Ten females recruited from Massachusetts General Hospital School of Nursing served as paid volunteers. Testing was divided into two sessions, one for the immediate recall of 76 lists of random digits in the forward order and one for the immediate recall of 76 similar lists in backward order. The sessions were separated by at least $24 \mathrm{~h}$. Half of the Ss received the forward order first, and half received the backward order first. Digit lists were also counterbalanced to insure that each appeared equally often under each order condition.

At the beginning of each session, the Ss were instructed that on each trial they would be read a short list of random digits to be recalled in either the forward or backward order, depending on the condition to which they had been assigned. Following the last digit of the list, a buzzer signaled them to begin recalling the digits. They were further instructed to recall the digits as fast as possible without sacrificing accuracy. The digit lists were read in a monotone at a $1 / \mathrm{sec}$ rate. The buzzer was sounded about $I$ sec after the last digit had been given. Several seconds following S's recall, she was cued to listen, and the next trial began. In all sessions, a 5 -min rest period was given between Trials 38 and 39 .

Each session began with the recall of a three-digit list. The list presented on each trial thereafter was one digit longer than the preceding one until S made an incorrect recall. An incorrectly recalled list was followed by a list one digit shorter in length. Each S's span was thereby determined by a method of adjustment.

All trials were recorded on a Roberts Model 1719 stereo tape recorder at $7 \frac{1}{2}$ ips. To measure the rate of recall, the completed tapes were played at $33 / 4$ ips into a Grass Model 5C polygraph with a fast paper speed of $7.2 \mathrm{~cm} / \mathrm{sec}$. This procedure allowed easy discrimination between responses and interresponse pauses. These two variables will be treated separately below. Only correctly recalled lists were included in the analyses. In addition, the first 10 trials under each condition were considered as practice and excluded.

\section{RESULTS}

The main findings are summarized in Fig. 1. The lower curve depicts the cumulative pause time between responses at each serial position in forward recall. An average pause of $676 \mathrm{msec}$ preceded the first response, and one of $131 \mathrm{msec}$ preceded each additional response thereafter. Each digit required an average of $197 \mathrm{msec}$ to be spoken. The equation of the line of best fit of the cumulative pause time was $\mathrm{RT}=.510+.131 \mathrm{SP}$, where SP indicates serial position in recall and RT denotes cumulative time between responses. The coefficient of determination was .99 . The upper curve in Fig. 1 shows the equivalent data for backward recall. Under this condition, the first digit was preceded by a pause of $1,294 \mathrm{msec}$ and each 